\title{
A Context-Aware Experience Sampling Tool
}

\author{
Stephen S. Intille \\ Charles Kukla \\ Isabel Iacono \\ Ling Bao \\ House_n: MIT Home of the Future Project \\ Massachusetts Institute of Technology \\ 1 Cambridge Center, 4FL, Cambridge MA 02142 USA \\ intille@mit.edu
}

\begin{abstract}
A new software tool for user-interface development and assessment of ubiquitous computing applications is available for $\mathrm{CHI}$ researchers. The software permits researchers to use common PDA mobile computing devices for experience sampling studies. The basic tool offers options not currently available in any other open-source sampling package. However, the tool also has one a completely new type of functionality: context-aware experience sampling. This feature permits researchers to acquire feedback from users only in particular situations that are detected by sensors connected to a mobile computing device.
\end{abstract}

\section{Keywords}

Context-aware, experience sampling, reflection, eliciting preferences, PDA, ubiquitous and mobile computing.

\section{THE PROBLEM}

User needs are typically elicited via personal or focus group interviews, site visits, and photographic and video analysis. Often, however, users know more than they say in a single or even several interviews [1]. As user interface design moves off the desktop and into the real world, two new challenges for designers emerge: (1) developing realistic task specifications that respond to the complexity of fastchanging, real world activities, and (2) evaluating new technologies in realistic contexts. Desktop computing applications can be designed and evaluated using controlled, laboratory observation because most user interface design has nothing to do with physical space [2]. Developers of ubiquitous and mobile computing applications for the home and workplace, however, currently lack a powerful and economical assessment toolset that accounts for user activity in a broader context. The behavior of the people and their response to technology is critically dependent upon the environment and context in which information is presented or requested.

The most popular assessment instruments in use today for studying the activities of people in natural settings are self report recall surveys, time diaries, direct field observation,

COPYRIGHT IS HELD BY THE AUTHOR/OWNER(S).

CHI 2003, APRIL 5-10, 2003, FT. LAUDERDALE, FLORIDA, USA.

ACM 1-58113-630-7/03/0004. and experience sampling. Self-report recall surveys suffer from recall and selective reporting biases - users can often not remember what they did. Time diaries, where users write down what they do during the day, are burdensome for the user. Although direct field observation can provide helpful qualitative and quantitative measures, it is costly, timeconsuming, and disruptive and therefore not practical for many design tasks. The experience sampling method (ESM) has been used primarily for time-use analysis [3] and only recently for interface design [4,5]. Subjects carry a beeper device that "samples" for information on some predetermined schedule. When the device beeps, subjects answer questions of interest to the researchers. With a sufficient number of subjects and samples, a statistical model of behavior can be generated. The ESM is less susceptible to subject recall errors than other self-report feedback elicitation methods [3], but it high sampling rates interrupt activities of interest and irritate subjects. Image-based experience sampling alleviates these problems but only in environments instrumented with cameras [4].

\section{CONTEXT-AWARE EXPERIENCE SAMPLING}

Multiple members of our group have research projects that would benefit from the use of a robust and flexible ESM tool. However, we found existing tools too inflexible difficult for users to use. We also needed a tool that would permit the sampling of the user directly via questioning as well as sampling from sensor devices worn or carried by the subject. For instance, we needed a tool that could acquire continuous sensor data from a device such as a GPS unit and also be used to acquire "ground truth" information directly from a subject about what the subject is doing.

We have, therefore, created new context-aware experience sampling software for PocketPC devices. The software can be used for standard experience sampling studies, but it has also been developed in a modular fashion that allows new context-sensing sensors and software to be plugged in. These sensors permit researchers to use context-sensitive sampling where specific questions are asked only when a user does a specific thing (e.g. is near the store). Contextawareness modules permit a researcher to acquire more information about the behavior or situation of interest by sampling only during or just after the activities of interest. This minimizes the interruption annoyance of the ESM technique. 

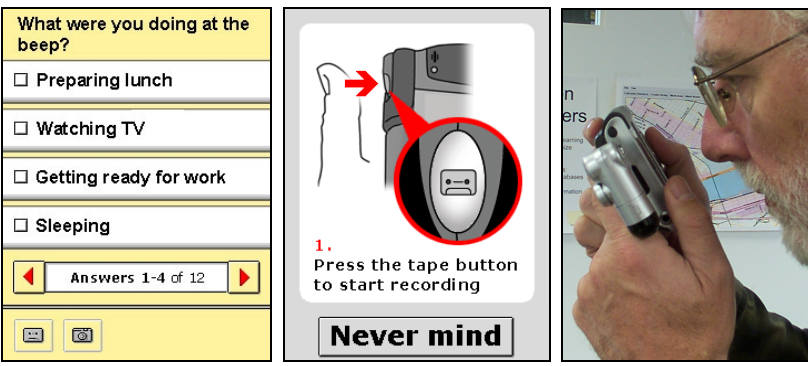

Figure 1: Screen shots of a multiple choice question screen and a tutorial screen for an audio note sample; sampling by taking a picture when a camera module is used.

\section{RELEASE 1 FEATURES}

We have used a participatory design process to create the interaction model for the ESM tool so that it suits both researcher and subject needs. Our goal has been to create software that permits a device to be handed to a subject at the start of a week with only a few minutes of instruction and then to be returned a week later with question and sensor data. The interface has been designed so that it is self-intuitive for middle-aged and older non-computer users.

The first release of the software includes capabilities for standard multiple choice question experience sampling using a time-sampled protocol. Researchers can load a new protocol by simply modifying a comma-delimited text file. Our software also includes protocol development flexibility not found in other open-source ESM software [6]. These include options for (1) chaining complex sequences of questions based upon particular question responses, (2) aggregation of questions to minimize user disruption in some situations, (3) study suppression during some events, (4) multiple choice and multiple response questions, (5) manual specification of precise query times for particular questions or question sequences, (6) flexible question recurrence patterns (by weeks, days, hours, minutes), and (7) bounded randomization ( $\mathrm{min} / \mathrm{max}$ time to next query). Further, researchers can allow users to leave answers via audio recording or, if the PDA has a camera plug-in, answer a question by taking a picture. The tool can therefore be used to combine the powerful techniques of ESM and photographic analysis [7]. The device includes just-in-time tutorials to aid subjects with some of the advanced functionality. Figure 1 shows two screen shots and a user taking a picture sample when the camera unit is plugged in.

The context-aware functionality of the first release includes the ability to sample based not only upon the standard timebased protocol but also upon a subject's location, as obtained by a GPS plug-in. Therefore, researchers can design studies that sample only when near a location of known interest.

\section{PLANNED EXTENSIONS AND APPLICATIONS}

Two additional context-aware extensions are planned within the year. These include (1) the ability to sample based upon heart-rate acquired via a wireless link from the
PDA to a chest strap heart monitor, and (2) the ability to query based upon particular activities identified using GPS context-recognition algorithms that have been created by our group. The software permits other sensor devices to be incorporated easily, and we invite researchers interested in doing so to contact us.

We have designed the tool to meet the needs of a set of ongoing and planned experiments in our laboratory. These include, among others, studies investigating (1) the development of algorithms that can detect various types of everyday activities automatically (e.g. walking, going to work, climbing stairs) using body-mounted accelerometers and a GPS plug-in, (2) how to create algorithms that automatically detect when people are transitioning between activities, and (3) how people interact in chance encounters in the workplace. Each study requires a different protocol that can be easily created by non-programmers. The results of some of our work on algorithms that automatically identify context that uses the tool will eventually be rolled back into the tool itself, providing future researchers with more context-specific triggers.

\section{CONCLUSION}

Context-aware experience sampling improves standard experience sampling and can be used by designers designing and evaluating ubiquitous and/or mobile computing applications. We have created this tool in response to needs created by ongoing and planned research projects at MIT. The tool is open source and the alpha version will be available via the web in February 2003 [6]. We encourage the CHI community to use the tool and to join our team to extend its experience sampling and context-aware sensing capabilities. This work is supported by the National Science Foundation and the House_n: MIT Home of the Future Research Consortium.

\section{REFERENCES}

1. Scheon, D.A. (1983) The Reflective Practitioner: How Professionals Think in Practice. New York, NY: Basic Books.

2. Mark, W. (1999) Turning Pervasive Computing into Mediated Spaces, IBM Systems Journal, 38(4).

3. Larson, R. and Csikszentmihalyi, M. (1983). The Experience Sampling Method. In H. T. Reis (Ed.), Naturalistic Approaches to Studying Social Interaction: New Directions for Methodology of Social and Behavioral Science. San Francisco, CA: Jossey-Bass.

4. Intille, S.S., Kukla, C. and Ma, X. (2002) Image-Based Experience Sampling and Reflection. Proceedings of CHI Extended Abstracts, pp. 738-9.

5. Hudson, J.M., Christensen, J., Kellogg, W.A. and Erickson, T. (2002) "I'd Be Overwhelmed, But It's Just One more Thing to Do:" Availability and Interruption in Research Management, Proceedings of CHI, pp. 97-102.

6. http://caes.sourceforge.net

7. Collier, J. and Collier, M. (1990) Visual Anthropology: Photography as a Research Method. Albuquerque, NM: University of New Mexico Press. 
